\title{
Double-Slit Electron Interference Experiment with Phase Modulation
}

Ken Harada ${ }^{1}$, Yoshio Takahashi ${ }^{2}$, Tetsuya Akashi ${ }^{2}$, Yoshimasa Ono ${ }^{1}$, Tetsuji Kodama ${ }^{3}$ and Shigeo Mori ${ }^{4}$

${ }^{1}$ RIKEN, Hatoyama, Saitama, Japan, ${ }^{2}$ Hitachi, Ltd., Hatoyama, Saitama, Japan, ${ }^{3}$ Meijo University, Nagoya, Aichi, Japan, ${ }^{4}$ Osaka Prefecture University, Sakai, Osaka, Japan

We have been conducting Young's double-slit experiments with electron beams using a 1.2 MV field emission electron microscope [1] with its excellent coherence, taking into account new viewpoints and techniques. In our previous studies, we tried clarifying which slit the electrons passed though using an asymmetric double-slit with different opening widths $[2,3]$ and a V-shaped double-slit with an opticallyzero-propagation distance from the slit to the detection plane [4]. However, we were unable to see electrons simultaneously in the interference state due to wave nature and the individual electron state due to particle nature.

In this paper, we studied how single electrons building up the interference fringes would shift their detected positions when electron wave phases were modulated after passed through the double-slit. We considered that each single electron as a wave changed its phase even when it traveled alone inside the microscope. To check this, we used the V-shaped double-slit employed in the previous experiment under the same optical system with the optically-zero-propagation distance. For the phase modulation we used optical-pass-length changes created by the electron beam tilt.

Figure 1 shows a schematic diagram of the optical system constructed in the 1.2-MV field-emission transmission electron microscope [1]. The V-shaped double-slit was placed at the object plane. The biprism I was placed at the image plane of the objective lens and the biprism II was placed at the crossover plane below the magnifying lens [5]. Interference conditions at the observation plane were controlled by applied voltages to the biprisms I and II. The phase modulation was controlled by using the deflector placed at an image plane under the double-slit.

Figure 2 shows interferograms with a relative phase difference $\varphi$; (a) $\varphi=0$, (b) $\varphi=\pi$, and (c) integrated image from 19 interferograms for one cycle of the phase modulation. We observed electrons as particles in sufficiently coherent states as shown in (a) and (b) with interference fringes displaced in accordance with the amount of phase modulation. Each single interferogram in (a) and (b) has interference fringes composed of single dot image of electrons, and interference fringes disappeared almost completely in the integrated image shown in (c). This is not because electron waves have become incoherent.

In conclusion, we succeeded in controlling the phase relations between two waves after passed though the $\mathrm{V}$-shaped slit. Even when each single interferogram has interference fringes composed of single dot image of electrons, the interference fringes disappeared in the integrated images. From these results, the relationship between the recording exposure time and beam coherence of the electron wave will be examined [6]. 


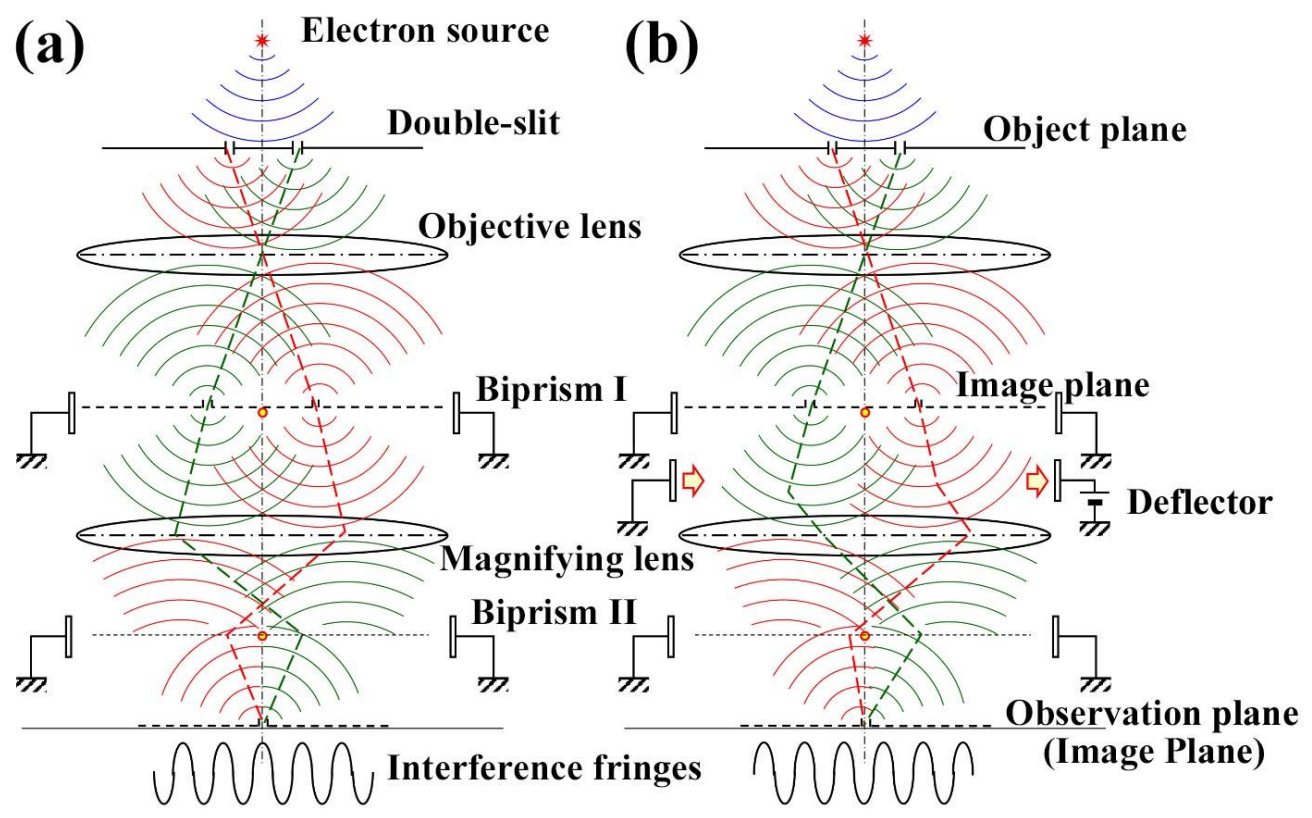

Figure 1. Schematic diagram of the interference experiment with phase modulation; (a) without phase difference, $\mathrm{Phi}=0$, (b) with phase difference, $\mathrm{Phi}=$ Pai.

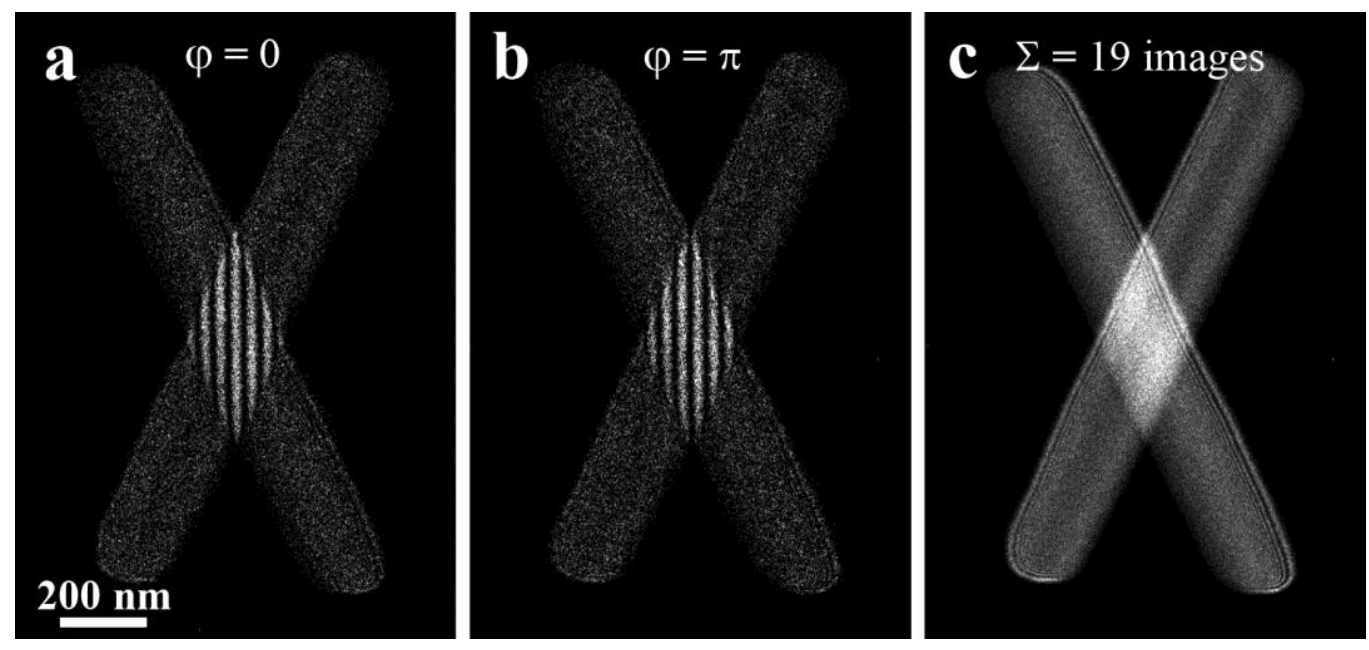

Figure 2. Interferograms by V-shaped double-slit with a relative phase difference Phi, (a) Phi = 0, (b) Phi $=$ Pai and (c) integrated image from 19 interferograms for one cycle of the phase modulation.

\section{References}

[1] T. Akashi et al., Appl. Phys. Lett., 106 (2015) 074101.

[2] K. Harada et al, Scientific Reports, 8 (2018) 1008.

[3] K. Harada et al., Microsc. Microanal., 24 (2018) 1966.

[4] K. Harada et al., Microsc. Microanal., 25 (2019) 944.

[5] K. Harada et al., Appl. Phys. Lett., 84 (2004) 3229.

[6] The authors thank Ms. K. Shimada of RIKEN for preparing the V-shaped slits. This work was supported by KAKENHI, Grant-in-Aid for Scientific Research ((B) 18H03475). 